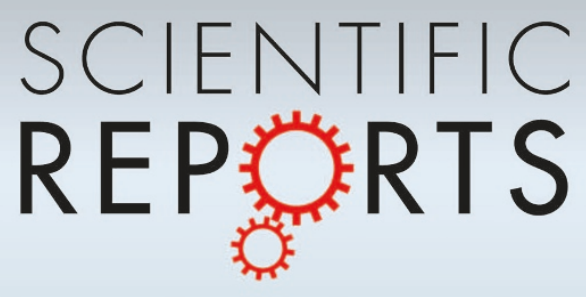

OPEN

SUBJECT AREAS:

ELECTRONIC DEVICES

CONDENSED-MATTER PHYSICS

SYNTHESIS AND PROCESSING

Received

14 October 2014

Accepted

9 December 2014

Published

12 January 2015

Correspondence and requests for materials should be addressed to

W.J.W. (wjweber@

utk.edu) or Y.Z.

(zhangy1@ornl.gov)

* Current address: School of Physics, State Key Laboratory of Crystal Materials and Key Laboratory of Particle Physics and Particle Irradiation (MOE), Shandong University, Jinan 250100, China.

\section{Synergy of elastic and inelastic energy loss on ion track formation in $\mathrm{SrTiO}_{3}$}

\author{
William J. Weber ${ }^{1,2}$, Eva Zarkadoula ${ }^{2}$, Olli H. Pakarinen2, Ritesh Sachan², Matthew F. Chisholm², \\ Peng Liu' ${ }^{*}$, Haizhou Xue', Ke Jin' \& Yanwen Zhang ${ }^{2}$
}

${ }^{1}$ Department of Materials Science and Engineering, University of Tennessee, Knoxville, TN 37996, USA, ${ }^{2}$ Materials Science and Technology Division, Oak Ridge National Laboratory, Oak Ridge, TN 37831, USA.

While the interaction of energetic ions with solids is well known to result in inelastic energy loss to electrons and elastic energy loss to atomic nuclei in the solid, the coupled effects of these energy losses on defect production, nanostructure evolution and phase transformations in ionic and covalently bonded materials are complex and not well understood due to dependencies on electron-electron scattering processes, electron-phonon coupling, localized electronic excitations, diffusivity of charged defects, and solid-state radiolysis. Here we show that a colossal synergy occurs between inelastic energy loss and pre-existing atomic defects created by elastic energy loss in single crystal strontium titanate $\left(\mathrm{SrTiO}_{3}\right)$, resulting in the formation of nanometer-sized amorphous tracks, but only in the narrow region with pre-existing defects. These defects locally decrease the electronic and atomic thermal conductivities and increase electron-phonon coupling, which locally increase the intensity of the thermal spike for each ion. This work identifies a major gap in understanding on the role of defects in electronic energy dissipation and electron-phonon coupling; it also provides insights for creating novel interfaces and nanostructures to functionalize thin film structures, including tunable electronic, ionic, magnetic and optical properties.

U nderstanding the response of materials to the separate and combined deposition of energy to electrons and to atomic nuclei is fundamentally important to accurately predict the behavior of materials in natural and man-made radiation environments, whether it is the decay of radioisotopes in natural minerals, radiation from fission or fusion in advanced nuclear reactors, the decay of nuclear waste, or cosmic radiation in space. Such understanding is also important to the use of electrons and ions in materials analysis, modification and functionalization, as well as to the use of ions for implantation doping, creating nanostructures and mimicking both natural and man-made radiation environments.

It is well-established that atomic-level defects are created by the transfer of energy to atomic nuclei in elastic collisions; however, the effects of inelastic energy loss to electrons on defect production and evolution ${ }^{1-3}$, nanostructure evolution ${ }^{4,5}$ and phase transformations ${ }^{6,7}$ are more complex and less understood. The importance of the effects of electronic energy loss and the coupling of electronic and atomic processes has become increasingly recognized in ionic and covalently bonded materials ${ }^{1,8-13}$. In these materials, the effects of electronic energy loss are generally observed to add linearly to defect production from atomic recoils ${ }^{1-5}$ or to compete linearly with defect production by inducing recovery processe ${ }^{6-9}$; however, ionization-enhanced mobility of point defects ${ }^{10,11}$ and nonlinear additivity of inelastic and elastic energy loss, ${ }^{3,12}$ lead to more complex behavior. Here we demonstrate an extraordinary synergy in ion-irradiated $\mathrm{SrTiO}_{3}$ between electronic energy loss and defects produced by atomic recoils - a clear case that irradiation-induced defects locally activate amorphous ion track formation processes that are not active in pristine crystals.

Due to its high dielectric constant and interesting electronic and optical properties, particularly when doped, $\mathrm{SrTiO}_{3}$ is a material with many applications in microelectronics ${ }^{14-17}$. Recently, $\mathrm{SrTiO}_{3}$ has been described as a critical foundational material in the area of functional oxide electronics ${ }^{18}$, as unexpected and emergent new magnetic properties are discovered. The heterointerface between amorphous and crystalline $\mathrm{SrTiO}_{3}$ can exhibit metallic conductivity ${ }^{19}$ that is similar to the transparent conductive layer produced on $\mathrm{SrTiO}_{3}$ by $300 \mathrm{eV} \mathrm{Ar}$ ion irradiation ${ }^{20}$. The amorphous structure of $\mathrm{SrTiO}_{3}$ is also found to exhibit polarity from a random network of partially aligned $\mathrm{TiO}_{6}$ octahedra ${ }^{21}$.

Irradiation of $\mathrm{SrTiO}_{3}$ at temperatures below $\sim 370 \mathrm{~K}$ with $0.1-1.0 \mathrm{MeV}$ ions leads to a crystalline-to-amorphous transformation due to the production and accumulation of atomic defects created by elastic energy transfer to atomic recoils ${ }^{22-24}$, and the temperature dependence of this transformation is controlled by defect recovery stages 
active below $350 \mathrm{~K}^{25}$. The chemical etch rate of the irradiationinduced amorphous structure is at least three orders of magnitude higher than the single crystal rate ${ }^{26}$, which has been used in combination of $1 \mathrm{MeV} \mathrm{Pt}$ ion irradiation to produce sub-100 nm patterns in $\mathrm{SrTiO}_{3}$ substrates. For ions at much higher energies, known as swift heavy ions, energy transfer to electrons dominate, and individual ion tracks, with diameters of $\sim 5$ to $6 \mathrm{~nm}$, have been reported for irradiation at $300 \mathrm{~K}$ with ions having electronic energy loss values from 20 to $50 \mathrm{keV} / \mathrm{nm}$ (92 MeV Xe to $2.0 \mathrm{GeV} \mathrm{U}$ ions) ${ }^{27,28}$. Swift heavy ion irradiation has also been shown to improve the photoelectrochemical activity of spin-coated $\mathrm{SrTiO}_{3}$ thin films ${ }^{29}$. The production of tracks, surface hillocks and property modification are attributed to a local thermal spike produced by the transfer of energy from the electrons to the atomic structure by electron-phonon coupling.

While these irradiation results demonstrate significant potential for modifying the structure and properties of $\mathrm{SrTiO}_{3}$ using electrons and ions, there is a lack of fundamental understanding regarding the thermal and irradiation stability of defects in $\mathrm{SrTiO}_{3}$ and the nature of ion tracks. Furthermore, only the effects of energy transfers to electrons have been investigated in the swift heavy ion studies ${ }^{27-29}$; while in ion irradiation studies of $\mathrm{SrTiO}_{3}$ significantly below the threshold for track formation, defect production and amorphization are primarily attributed to elastic energy transfers to atomic nuclei $^{22-24}$, despite the comparable electronic energy loss of the ions. In this paper, we reveal the effect of atomic defects, produced by elastic energy loss, on the local response of $\mathrm{SrTiO}_{3}$ to electronic energy loss below the threshold for amorphous track formation.

\section{Results}

A critical research challenge is to understand the response of materials to the coupled or combined effects of elastic and inelastic energy loss; however, when these occur simultaneously, it can be difficult to quantify the individual effect or contribution. Here we conduct a separate effects study, in which single crystals of $\mathrm{SrTiO}_{3}$ are predamaged with $900 \mathrm{keV} \mathrm{Au}$ ions at $300 \mathrm{~K}$ to produce a low concentration of defects from elastic energy loss, with a fractional peak disorder of $\sim 0.07$ at a depth of $\sim 90-95 \mathrm{~nm}$ as shown in Fig. 1a. Both undamaged and pre-damaged single crystals are then subsequently irradiated to different ion fluences with $21 \mathrm{MeV} \mathrm{Ni}$ ions, which have a high electron energy loss of 9.5 to $10 \mathrm{keV} / \mathrm{nm}$ across the pre-damaged thickness (Fig. 1a), but a very low nuclear energy loss of $0.09 \mathrm{keV} / \mathrm{nm}$ (not shown). The damage evolution in these samples is characterized by Rutherford backscattering spectrometry along a channeling direction (RBS/C) and scanning transmission electron microscopy (STEM).

Irradiation of the pristine, undamaged $\mathrm{SrTiO}_{3}$ with $21 \mathrm{MeV} \mathrm{Ni}$ ions up to fluences of 0.2 ions $/ \mathrm{nm}^{2}$ did not result in any measurable
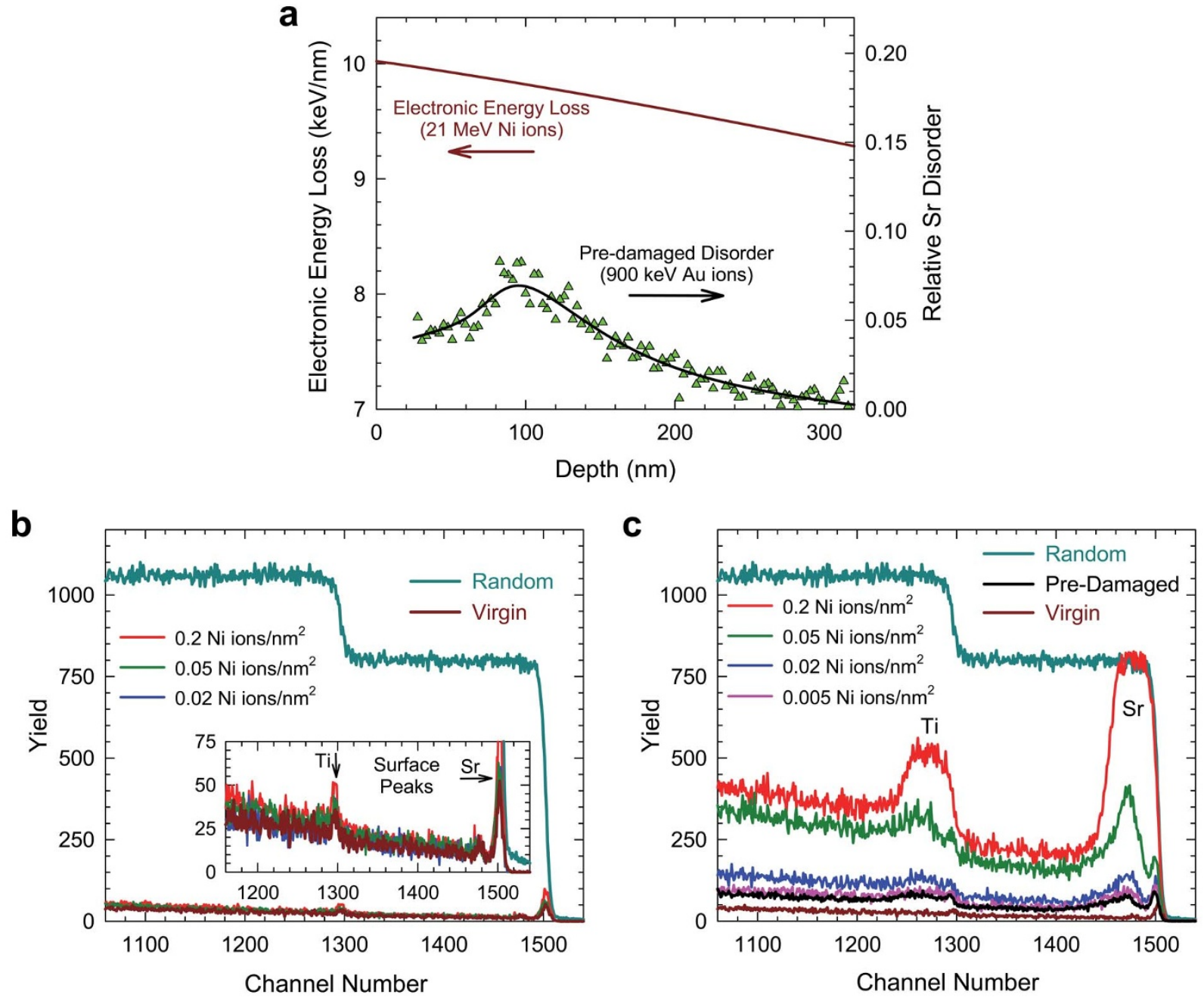

Figure 1 Pre-damaged state and effect of electronic energy loss on undamaged and pre-damaged $\mathrm{SrTiO}_{3}$. (a) Damage profile introduced by $900 \mathrm{keV}$ $\mathrm{Au}$ ions to a fluence of 0.13 ions $/ \mathrm{nm}^{2}$ and electronic energy loss profile for $21 \mathrm{MeV} \mathrm{Ni}$ ions. Rutherford backscattering spectra along [100] direction: (b) undamaged $\mathrm{SrTiO}_{3}$ irradiated with $21 \mathrm{MeV} \mathrm{Ni}$ ions to fluences up to 0.2 ions/nm $\mathrm{nm}^{2}$; and (c) pre-damaged $\mathrm{SrTiO}_{3}$ irradiated with $21 \mathrm{MeV} \mathrm{Ni}$ ions to fluences up to 0.2 ions $/ \mathrm{nm}^{2}$ (spectrum for ion fluence of $0.001 \mathrm{Ni}$ ions $/ \mathrm{nm}^{2}$ not shown). 
increase in the backscattering yield (Fig. 1b), indicating insensitivity of the undamaged state to defect production and track formation from the inelastic and elastic energy losses of the $21 \mathrm{MeV} \mathrm{Ni}$ ions. Based on the low elastic energy losses and the typical doses ${ }^{24}$ to amorphize $\mathrm{SrTiO}_{3}$, it would be expected that an ion fluence on the order of 100 ions $/ \mathrm{nm}^{2}$ would be required to amorphize $\mathrm{SrTiO}_{3}$ with $21 \mathrm{MeV} \mathrm{Ni}$ ions at a depth of $100 \mathrm{~nm}$. Thus, it was quite unexpected when we discovered that irradiation of the pre-damaged state with $21 \mathrm{MeV} \mathrm{Ni}$ ions resulted in a rapid and dramatic increase in the backscattering yield at much lower ion fluences, 0.001 to 0.2 ions/ $\mathrm{nm}^{2}$ (Fig. 1c); this surprising result demonstrates a highly synergistic effect of the pre-existing defect structure on the local response to inelastic energy loss to electrons. This is further revealed by the detailed analysis of the RBS/C spectra (Fig. 2a) that shows the evolution of the relative disorder as a function of depth. The results indicate that the fully amorphous state (relative disorder $=1.0$ ) is achieved over a depth from $\sim 30$ to $120 \mathrm{~nm}$ at a relatively low ion fluence of $0.2 \mathrm{Ni}$ ions $/ \mathrm{nm}^{2}$; the local elastic energy loss dose for this ion fluence is less than 0.0015 displacements per atom (dpa), which is 2-3 orders of magnitude lower than that required to produce a fully amorphous state in $\mathrm{SrTiO}_{3}$ by elastic collision processes ${ }^{24}$. The very rapid increase in disorder at low ion fluences follows trends normally seen for the formation of amorphous ion tracks by swift heavy ions in complex oxides ${ }^{28,30}$; here however, the results suggest that the amorphous ion tracks are only formed in the pre-damaged layer and end abruptly at a depth of $\sim 160-170 \mathrm{~nm}$, which is unlike continuous track formation by swift heavy ions.

These results demonstrate that the introduction of a low concentration of defects greatly enhanced the sensitivity of $\mathrm{SrTiO}_{3}$ to the effects of electronic energy loss and, hence, locally lowered the threshold for amorphous track formation. Somewhat similar behavior has been reported for track formation in pre-damaged GaAs and InP following irradiation with $573 \mathrm{MeV}$ Au ions and attributed to an increase in electron-phonon coupling from pre-existing defects ${ }^{12}$. A direct impact model for amorphization (see Supplementary information) is fit to the increase in disorder, measured at several different depths, with increasing ion fluence (see Supplementary Fig. 1). Based on these model fits, the effective amorphous track diameter has been determined as a function of depth, as shown in Fig. 2b. The track diameter ranges from about $1.2 \mathrm{~nm}$, corresponding to an initial relative disorder level of 0.04 , to a value of about $2.2 \mathrm{~nm}$, corresponding to the initial disorder level of 0.07 at the disorder peak. These track

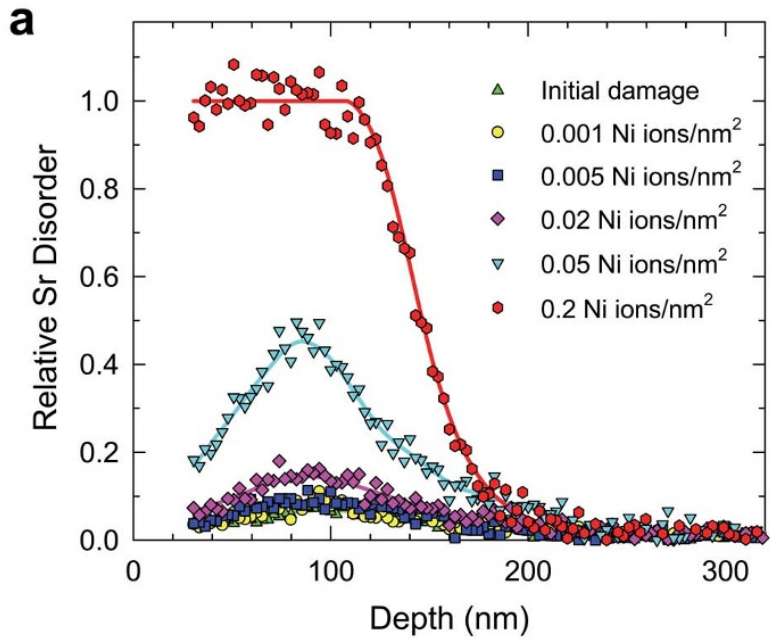

diameters are much smaller than the tracks diameters (5-6 nm) determined for $\mathrm{SrTiO}_{3}$ irradiated with swift heavy ions having electronic energy loss values ranging from 20 to $50 \mathrm{keV} / \mathrm{nm}^{28,27}$.

Scanning transmission electron microscopy. STEM with high angle annular dark field (HAADF) imaging was used to characterize the irradiated samples. The HAADF image in Fig. 3 clearly shows a circular ion track with an amorphous core diameter of $\sim 2 \mathrm{~nm}$ following $21 \mathrm{MeV} \mathrm{Ni}$ ion irradiation to 0.02 ions $/ \mathrm{nm}^{2}$, which is consistent with the experimentally derived range of track diameters shown in Fig. 2b. This HAADF result is in distinct contrast to prior work ${ }^{28}$ where only recrystallized tracks could be imaged. Because of the sensitivity of amorphous layers in $\mathrm{SrTiO}_{3}$ to epitaxial recrystallization by electron-beam irradiation ${ }^{24}$, some initial recrystallization of the amorphous track in Fig. 3 cannot be ruled out; however, no change in track diameter was observed after continuous imaging, as illustrated in Supplementary Video 1, suggesting that these amorphous tracks are relatively stable to highenergy electron beam irradiations, as demonstrated here for $200 \mathrm{keV}$ electrons.

Simulation. Large scale molecular dynamics (MD) simulations (7 million atoms) were performed using a simulation cell without any defects and a simulation cell containing the equivalent of $\sim 1.5 \%$ Frenkel defects. In the case of the defective simulation cell, Frenkel pairs were introduced randomly in the system, which was then relaxed under constant pressure and temperature. The radial energy deposition profiles for the average electronic energy loss $(9.74 \mathrm{keV} / \mathrm{nm})$ by the $21 \mathrm{MeV} \mathrm{Ni}$ ions over a depth of $25 \mathrm{~nm}$ in the perfect crystal and in the pre-damaged system were based on an inelastic thermal spike model ${ }^{30}$ using input derived from the two-temperature model (see Supplementary information). To account for increased scattering of electrons and phonons from defects in the two-temperature model, the thermal conductivities of the electronic and atomic systems for the pre-damaged system were assumed to be an order of magnitude smaller than the perfect crystal system, and the electron phonon-coupling constant for the pre-damaged system assumed to be $40 \%$ larger than the value calculated for the perfect crystal. The thermal spike from the energy deposition profile (Fig. 4a) for the perfect crystal did not produce clearly discernible defects in the simulation cell without defects, as illustrated in Supplementary Video 2. Similarly, the

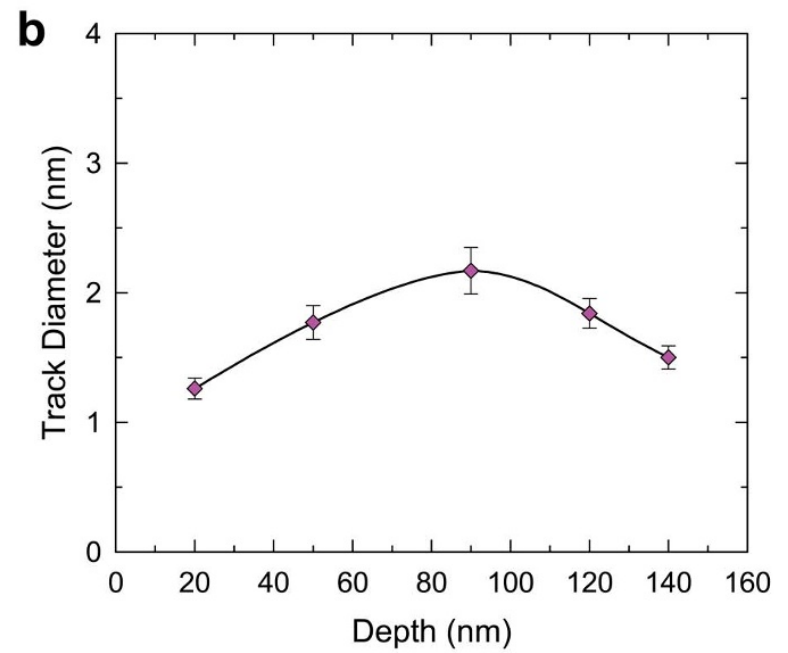

Figure $2 \mid$ Amorphous track formation from electronic energy loss in pre-damaged $\mathrm{SrTiO}_{3}$. (a) Relative Sr disorder determined from Rutherford backscattering spectra (Fig. 1c) as a function of depth. (b) Track diameter determined at different depths from analysis of increase in disorder in (a) with ion fluence (error bars based on one standard deviation in track cross section from fit of direct impact model to change in disorder at given depth; solid line is spline fit). 


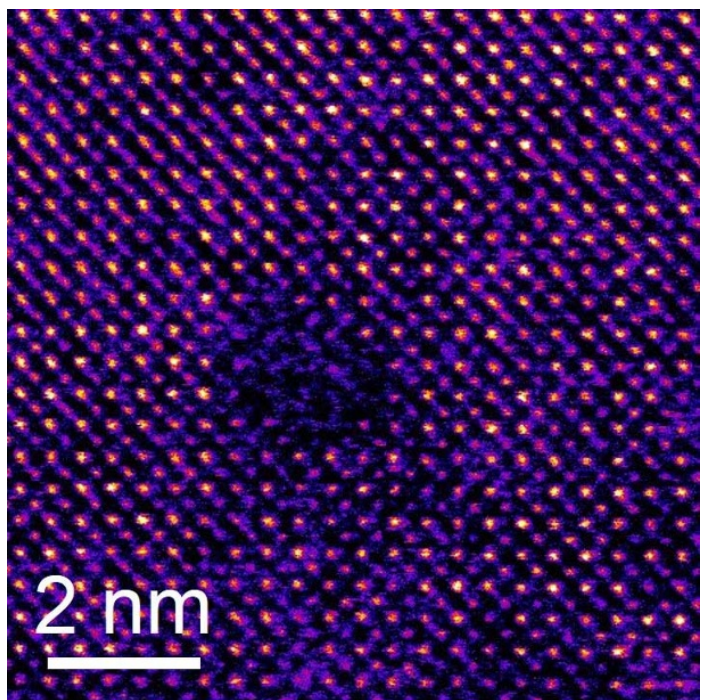

Figure $3 \mid$ High angular annular dark field image of ion track in predamaged $\mathrm{SrTiO}_{3}$. High resolution atomic image from STEM characterization with $200 \mathrm{keV}$ electrons showing central amorphous core of ion track produced by irradiation with $21 \mathrm{MeV} \mathrm{Ni}$ ions to a fluence of 0.02 ions $/ \mathrm{nm}^{2}$

thermal spike from the energy deposition profile calculated for the pre-damaged state (Fig. 4a) did not produce an amorphous track in the simulation cell without defects, although in this case a few isolated residual defects were produced. However, in the simulation cell containing a random distribution of Frenkel defects, the corresponding thermal spike (Fig. 4a) produced an amorphous track of $\sim 2 \mathrm{~nm}$ diameter, as shown in Fig. $4 \mathrm{~b}$, consistent with the experimental results shown in Figs. $2 b$ and 3. The track formation process is illustrated in Supplementary Video 3 , which shows that track formation is continuous through the thickness of the defective simulation cell. These results clearly demonstrate that the pre-existing defects, not only affect the energy deposition profile from the two-temperature model, but also play a critical role in track evolution in the MD simulations.

The distortions of both the $\mathrm{Sr}$ column of atoms and the $\mathrm{TiO}_{6}$ octahedra along the [100] direction are evident within the amorphous track (Fig. 4b), consistent with the structure of amorphous $\mathrm{SrTiO}_{3}$ determined by $\mathrm{x}$-ray absorption fine structure measurements ${ }^{21}$. These

a

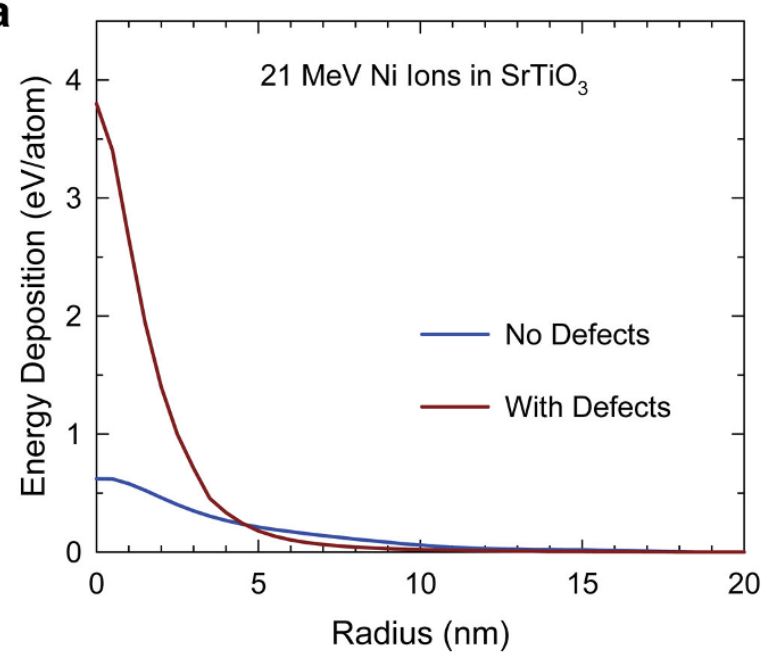

results confirm that the introduction of cascade-like defects sensitizes $\mathrm{SrTiO}_{3}$ to amorphous track formation at relative low values of electronic energy loss. While the MD simulations are qualitatively consistent with our experimental observations and expectations that atomic scale defects should decrease thermal conductivities and increase electron-phonon coupling, the magnitude of the changes are not well known or readily predicted. More research is clearly needed on the effects of defects and their concentration on electronic and atomic conductivities and electron-phonon coupling, and first principle calculations could provide valuable contributions in this area. Likewise, more experimental research is needed to determine the thresholds, both in initial defect concentration and energy loss, for this synergistic coupling between defects and electronic energy loss.

In conclusion, these results demonstrate that a low concentration of defects introduced by elastic collisions in $\mathrm{SrTiO}_{3}$ interacts synergistically with the inelastic electronic energy loss to produce amorphous tracks; whereas, tracks are not produced under similar irradiation conditions in the absence of defects. This synergistic effect effectively decreases the electronic energy loss threshold for amorphous track formation to much lower values than previously suggested by swift heavy ion irradiations, which may necessitate the reinterpretation of data on irradiation induced amorphization in $\mathrm{SrTiO}_{3}$ and other materials at intermediate energies. Since such tracks can be produced with intermediate energy ions, which are widely available in research and industry, this work provides a new pathway for the exploitation of such tracks in $\mathrm{SrTiO}_{3}$ substrates and thin films. Due to differences in thermal stabilities of the low concentration of defects and the amorphous tracks ${ }^{23,25}$, thermal annealing may lead to isolated amorphous tracks or pillars embedded in a highly crystalline matrix. Based on what is currently known on the chemical and physical properties of these nanoscale amorphous tracks and their interface structures in $\mathrm{SrTiO}_{3}$, there are numerous possible impacts; these include: 1) selective chemical etching of tracks or patterns created by ion-beam writing in 3D nanofabrication; 2) creating amorphous pillars or patterns of well-defined length from the surface or buried in the matrix; 3 ) using the heterointerface structure or further modifying the interface structure to create new one-dimensional electronic and magnetic functionalities; and 4) utilize the unique polarity of amorphous $\mathrm{SrTiO}_{3}$ to create one-dimensional polarity normal to surface in controlled patterns.

\section{Methods}

Irradiation procedure. The [100] oriented single crystal $\mathrm{SrTiO}_{3}$ samples were irradiated at $7^{\circ}$ off the [100] direction with $900 \mathrm{keV}$ Au ions to a fluence of 0.13 ions/

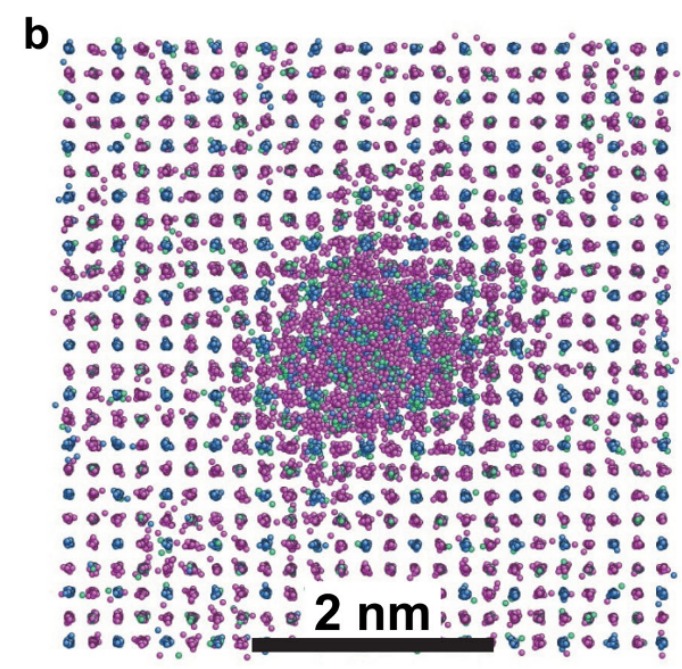

Figure $4 \mid \mathrm{MD}$ simulation of ion track formation in $\mathrm{SrTiO}_{3}$ containing 1.5\% Frenkel pairs. (a) Energy deposition profiles for $21 \mathrm{MeV} \mathrm{Ni}$ ions in $\mathrm{SrTiO}_{3}$ with no defects and with 1.5\% defects. (b) Atomic plot along the [001] direction showing crystal structure, defects, and amorphous track after a thermal spike characteristic of a $21 \mathrm{MeV} \mathrm{Ni}$ ion in the simulation cell with $1.5 \%$ defects (blue $=\mathrm{Sr}$, green $=\mathrm{Ti}$, and purple $=\mathrm{O}$ ). 
$\mathrm{nm}^{2}$ at $300 \mathrm{~K}$ to produce a stable concentration of defects at a low relative disorder level of 0.07 at the damage peak (Fig. 1a). After initial characterization of the disorder distribution by RBS/C along the [100] direction, the samples were irradiated with $21 \mathrm{MeV} \mathrm{Ni}$ ions at $7^{\circ}$ off the [100] direction. The electronic and nuclear energy losses for $21 \mathrm{MeV} \mathrm{Ni}$ ions and the atomic displacements by $900 \mathrm{keV} \mathrm{Au}$ ions were determined as a function of depth in $\mathrm{SrTiO}_{3}$ using the Stopping and Range of Ions in Matter (SRIM) code ${ }^{31,32}$. The electronic energy loss by $21 \mathrm{MeV} \mathrm{Ni}$ ions in the surface region of $\mathrm{SrTiO}_{3}$ is shown in Fig. 1a. The irradiations and $\mathrm{RBS} / \mathrm{C}$ were conducted at the Ion Beam Materials Laboratory at the University of Tennessee ${ }^{33}$.

RBS/C. This technique is sensitive to atomic displacements along major crystallographic channeling directions, where the probing He ions are backscattered from interstitials or disordered atoms, and is used to quantitatively determine irradiation-induced damage in crystalline samples. The undamaged and predamaged $\mathrm{SrTiO}_{3}$, as well as the Ni-irradiated undamaged and pre-damaged samples, were characterized by RBS/C along the [100] direction (Figs. 1b,c). The RBS/C spectra were analyzed using an iterative procedure ${ }^{34}$ to determine the relative disorder as a function of depth (Fig. 2a)

STEM. HAADF imaging was performed on various samples in a $5^{\text {th }}$ order aberration corrected scanning transmission electron microscope (Nion UltraSTEM200) operating at $200 \mathrm{KV}$. Detector with an inner angle of $65 \mathrm{mrad}$ was used to collect electrons for HAADF imaging. The electron probe current and the exposure time/ pixel for imaging in the experiment was $16 \mathrm{pA}$ and $5 \mu \mathrm{sec}$ to minimize the electron beam induced modification.

Molecular dynamics. The MD simulations were run using the DL_POLY code ${ }^{35}$. The heat that was transferred to the atomic system due to the electronic excitations was calculated using an inelastic thermal spike model $^{30,36}$ and was used as input in the MD simulations. In the inelastic thermal spike model, the heat exchange between the lattice and the electronic system is described by a set of coupled heat diffusion equations for the electronic and the atomic system. We used the McCoy et al. potential $^{37}$, joined to short-range repulsive ZBL potentials ${ }^{32}$. The ZBL potential was used for all pair interactions. The cylindrical thermal spike was deposited along the $\mathrm{z}$ direction through the thickness of the simulation cell, and a boundary layer with a thickness of about $1 \mathrm{~nm}$ in the $\mathrm{x}$ and $\mathrm{y}$ directions was connected to a thermostat at $300 \mathrm{~K}$ to emulate the effect of energy dissipation into the sample and to allow heat to dissipate from the track region. The simulations were run in a constant energy and volume ensemble, preceded by relaxation of the systems under constant pressure and temperature. The value of the electronic energy loss used in the inelastic thermal spike model for the simulations is $9.74 \mathrm{keV} / \mathrm{nm}$. The thermal conductivity of the atomic and electronic subsystems in the pre-damaged system was decreased by an order of magnitude compared to the values for the perfect crystal. The electron-phonon coupling constant was calculated, as described elsewhere ${ }^{36}$, to be $4.3 \times 10^{18} \mathrm{~W} \mathrm{~m}^{-3}$ $\mathrm{K}^{-1}$ for the perfect crystal, and a value $40 \%$ larger, $6.0 \times 10^{18} \mathrm{~W} \mathrm{~m}^{-3} \mathrm{~K}^{-1}$, was assumed for the pre-damaged sample.

1. Toulemonde, M. et al. Synergy of nuclear and electronic energy losses in ionirradiation processes: the case of vitreous silicon dioxide. Phys. Rev. B 83, 054106 (2011).

2. Thomé, L. et al. Combined effects of nuclear and electronic energy losses in solids irradiated with dual-ion beam. Appl. Phys. Lett. 102, 141906 (2013).

3. Sall, M. et al. Synergy between electronic and nuclear energy losses for color center creation in AlN. EPL 102, 26002 (2013).

4. Zhang, Y. et al. The effect of electronic energy loss on irradiation-induced grain growth in nanocrystalline oxides. Phys. Chem. Chem. Phys. 16, 8051-8059 (2014).

5. Wang, Y. Y. et al. Energy deposition by heavy ions: Additivity of kinetic and potential energy contributions on hillock formation in $\mathrm{CaF}_{2}$. Sci. Rep. 4, 5742 (2014).

6. Debelle, A. et al. Combined experimental and computational study of the recrystallization process induced by electronic interactions of swift heavy ions with silicon carbide crystals. Phys. Rev. B 86, 100102 (2012).

7. Weber, W. J., Zhang, Y., Xiao, H. Y. \& Wang, L. M. Dynamic recovery in silicateapatite structures under irradiation and implications for long-term immobilization of actinides. RSC Advances 2, 595-604 (2012).

8. Zhang, Y. et al. Competing effects of electronic and nuclear energy loss on microstructural evolution in ionic-covalent materials. Nucl. Instrum. Methods. Phys. Res. B 327, 33-43 (2014).

9. Backman, M. et al. Cooperative effect of electronic and nuclear stopping on ion irradiation damage in silica. J. Phys. D: Appl. Phys. 45, 505305 (2012).

10. Zinkle, S. J., Skuratov, V. \& Hoelzer, D. T. On the conflicting roles of ionizing radiation in ceramics. Nucl. Instrum. Methods Phys. Res. B 191, 758-766 (2002).

11. Kinoshita, C., Yasuda, K., Matsumura, S. \& Shimada, M. Effects of simultaneous displace and ionizing radiations and effects of electric field on radiation damage in ionic crystals. Metall. Mater. Trans. A 35, 2257-2266 (2004).

12. Kamarou, A., Wesch, W. \& Wendler, E. Radiation damage in InP, InSb, GaAs, $\mathrm{GaP}, \mathrm{Ge}$, and Si due to fast ions. Phys. Rev. B 78, 054111 (2008).

13. Duffy, D. M., Daraszewicz, S. L. \& Mulroue, J. Modelling the effects of electronic excitations in ionic-covalent materials. Nucl. Instrum. Methods Phys. Res. B 277, 21-27 (2012).
14. Wilk, G. D., Wallace, R. M. \& Anthony, J. M. High-k gate dielectrics: Current status and materials properties considerations. J. Appl. Phys. 89, 5243-5275 (2001).

15. Haeni, J. H. et al. Room-temperature ferroelectriciy in strained $\mathrm{SrTiO}_{3}$. Nature 430, 758-761 (2004).

16. Kosola, A., Putkonen, M., Johansson, L. S. \& Niinistö, L. Effect of annealing in processing strontium titanate thin films by ALD. Appl. Surf. Sci. 211, 102-112 (2003).

17. Spinelli, A., Torija, M. A., Liu, C., Jan, C. \& Leighton, C. Electronic transport in doped $\mathrm{SrTiO}_{3}$ : Conduction mechanisms and potential applications. Phys. Rev. B 81, 1555110 (2010)

18. Rice, W. D. et al. Persistent optically induced magnetism in oxygen-deficient strontium titanate. Nature Materials 13, 481-487 (2014)

19. Chen, Y. et al. Metallic and insulating interfaces of amorphous $\mathrm{SrTiO}_{3}$-based oxide heterostructures. Nano Lett. 11, 3774-3778 (2011).

20. Reagor, D. W. \& Butko, V. Y. Highly conductive nanolayers on strontium titanate produced by preferential ion-beam etching. Nature Materials 4, 593-596 (2005).

21. Frenkel, A. I. et al. Origin of polarity in amorphous $\mathrm{SrTiO}_{3}$. Phys. Rev. Lett. 99, 215502 (2007).

22. Oyoshi, K., Hishita, S. \& Haneda, H. Study of ion beam induced epitaxial recrystallization of $\mathrm{SrTiO}_{3}$. J. Appl. Phys. 87, 3450-3456 (2000).

23. Meldrum, A., Boatner, L. A., Weber, W. J. \& Ewing, R. C. Amorphization and recrystallization of $\mathrm{ABO}_{3}$ oxides. J. Nucl. Mater. 300, 242-254 (2002)

24. Zhang, Y. et al. Ion-induced damage accumulation and electron-beam-enhanced recrystallization in $\mathrm{SrTiO}_{3}$. Phys. Rev. B 72, 094112 (2005)

25. Weber, W. J. et al. Ion-beam-induced defects and defects interactions in perovskite-structure titanates, in Defects and Surface-Induced Effects in Advanced Perovskites (eds. Borstel, G., Krumins, A. \& Millers, D.D.) 317-328 (Kluwer Academic Publishers, Dordrecht, , The Netherlands, 2000).

26. Matsuura, N. et al. Ultrahigh-density, nonlithographic, sub-100 nm pattern transfer by ion implantation and selective chemical etching. Appl. Phys. Lett. 81, 4826-4828 (2002).

27. Li, W. et al. Effect of doping of the radiation response of conductive $\mathrm{Nb}-\mathrm{SrTiO}_{3}$. Nucl. Instrum. Methods Phys. Res. B 302, 40-47 (2013).

28. Grygiel, C. et al. Online in situ diffraction setup for structural modification studies during swift heavy ion irradiation. Rev. Sci. Instrum. 83, 013902 (2012).

29. Solanki, A. et al. Irradiation-induced modifications of PEC response - A case study of $\mathrm{SrTiO}_{3}$ thin films irradiated by $120 \mathrm{MeV} \mathrm{Ag}$ (ons. Int. J. Hydrogen Energy 36, 5236-5245 (2011).

30. Toulemonde, M. et al. Experimental phenomena and thermal spike model description of ion tracks in amorphisable ionorganic insulators. Mat. Fys. Medd K. Dan. Vidensk. Selsk. 52, 263-292 (2006).

31. Ziegler, J. F., Biersack, J. P. \& Ziegler, M. D. SRIM - The Stopping and Range of Ions in Matter. (SRIM Co., Chester, MD, USA, 2008).

32. Ziegler, J. F., Biersack, J. P. \& Littmark, U. The Stopping and Range of Ions in Matter (New York, Pergamon, 1985).

33. Zhang, Y. et al. New ion beam materials laboratory for effective investigation of materials modification and irradiation effects. Nucl. Instrum. Methods Phys. Res. B 338, 19-30 (2014).

34. Zhang, Y. et al. Response of Strontium Titanate to Ion and Electron Irradiation. J. Nucl. Mater. 389, 303-310 (2009)

35. Todorov, I. T., Smith, W., Trachenko, K. \& Dove, M. T. DL_POLY 3: New dimensions in molecular dynamics simulations via massive parallelism. J. Mater. Chem. 16, 1911-1918 (2006).

36. Meftah, A. et al. Experimental determination of track cross-section in $\mathrm{Gd}_{3} \mathrm{Ga}_{5} \mathrm{O}_{12}$ and comparison to the inelastic thermal spike model applied to several materials. Nucl. Instrum. Methods Phys. Res. B 237, 563-574 (2005).

37. McCoy, M. A., Grimes, R. W. \& Lee, W. E. Phase stability and interfacial structures in the $\mathrm{SrO}-\mathrm{SrTiO}_{3}$ system. Philos. Mag. A 75, 833-846 (1997).

\section{Acknowledgments}

This work was supported by the U.S. Department of Energy, Office of Basic Energy Sciences, Materials Sciences and Engineering Division. This research used resources of the National Energy Research Scientific Computing Center, supported by the Office of Science, US Department of Energy under Contract No.DEAC02-05CH11231.

\section{Author contributions}

W.J.W. and Y.Z. conceived the experiments and performed the data analysis. P.L., H.X. and K.J. performed the irradiations and RBS/C measurements. R.S. and M.F.C. performed the scanning transmission electron microscopy analysis. E.Z. and O.H.P. performed two-temperature model calculations and MD simulations. W.J.W., Y.Z., R.S. and E.Z. wrote the paper in consultation with all authors.

\section{Additional information}

Supplementary information accompanies this paper at http://www.nature.com/ scientificreports

Competing financial interests: The authors declare no competing financial interests.

How to cite this article: Weber, W.J. et al. Synergy of elastic and inelastic energy loss on ion track formation in $\mathrm{SrTiO}_{3}$. Sci. Rep. 5, 7726; DOI:10.1038/srep07726 (2015). 
cc)(i) (2) This work is licensed under a Creative Commons Attribution-NonCommercialShareAlike 4.0 International License. The images or other third party material in this article are included in the article's Creative Commons license, unless indicated otherwise in the credit line; if the material is not included under the Creative
Commons license, users will need to obtain permission from the license holder in order to reproduce the material. To view a copy of this license, visit http:// creativecommons.org/licenses/by-nc-sa/4.0/ 